\title{
Sepsis Due to Bloodstream Ralstonia mannitolilytica Infection After Surgical Treatment of Perianal Abscess: A Case Report
}

\author{
Jiamin $T u^{\prime}$ \\ Lei Tu' \\ Yinping Jiang ${ }^{2}$ \\ Honghao $\mathrm{Fu}^{\mathrm{I}}$ \\ Zijuan $\mathrm{Mai}^{3}$ \\ Xianglin $\mathrm{Wu}^{4}$ \\ Bing $X u^{\prime}$
}

'Department of Proctology, University of Chinese Academy of Science Shenzhen Hospital (GuangMing), Shenzhen, 518100, People's Republic of China; ${ }^{2}$ Department of Chinese Medicine, University of Chinese Academy of Science Shenzhen Hospital (GuangMing), Shenzhen, 518100, People's Republic of China; ${ }^{3}$ Department of Medical Record Statistics Section, University of Chinese Academy of Science Shenzhen Hospital (GuangMing), Shenzhen, 518100, People's Republic of China; ${ }^{4}$ Department of Laboratory, University of Chinese Academy of Science Shenzhen Hospital (GuangMing), Shenzhen, 518100, People's Republic of China
Correspondence: Jiamin Tu

Department of Proctology, University of Chinese Academy of Science Shenzhen Hospital (GuangMing), No. 4253 Songbai Road, Guangming District, Shenzhen, 518100 , People's Republic of China Tel +86I5889790570

Email jiajiabaxia@।26.com
Background: Ralstonia mannitolilytica can cause opportunistic infections. Reports on this pathogen identified in the bloodstream are rare worldwide, especially in China.

Case Description: We describe a 48-year-old man who developed sepsis due to bloodstream Ralstonia mannitolilytica infection after surgery for a perianal abscess. His condition deteriorated into multiple organ dysfunction syndromes until susceptible antibiotics (ceftriaxone and levofloxacin) were administrated based on the drug sensitivity test results. The patient had a satisfactory recovery with no complications during a 6-month follow-up period. Conclusion: Ralstonia mannitolilytica blood-borne infection in patients evolves rapidly. The inconsistent sensitivity to antibiotics makes timely treatment difficult and can lead to serious complications. We report the clinical presentations and treatment outcomes for this patient here to remind clinicians about this rare opportunistic pathogen and to highlight the importance of bacterial culture, especially for immunocompromised patients.

Keywords: Ralstonia mannitolilytica, sepsis, shock, perianal abscess, multiple organ dysfunction syndrome

\section{Introduction}

The genus Ralstonia comprises a group of Gram-negative bacteria commonly found in the environments. Three species, Ralstonia pickettii, Ralstonia insidiosa, and Ralstonia mannitolilytica, have been identified as opportunistic human pathogens. ${ }^{1}$ Among them, $R$. pickettii is the most frequently reported pathogen, and only a limited number of infections are attributed to $R$. insidiosa and $R$. mannitolilytica. The clinical importance of these two latter species is probably underestimated because their biochemical patterns are similar to those of $R$. pickettii, making it difficult to differentiate them from R. pickettii based only on the results of conventional microbiological tests. ${ }^{2,3}$ Several studies have reported problematic results when identifying Ralstonia species based on commercially available tests. ${ }^{4-6}$ Here, we describe a rare case with rapidly progressive sepsis and multiple organ dysfunction syndromes (MODS) caused by $R$. mannitolilytica after surgical treatment for perianal abscess. The pathogen, $R$. mannitolilytica, was identified using the Bruker MALDI-TOF mass spectrometry system.

\section{Case Description}

A 48-year-old man was admitted to the Proctology Department of the University of Chinese Academy of Science Shenzhen Hospital, China on August 22nd, 2020 due 
to perianal abscess. He had a medical history of syphilis but had not received treatment due to penicillin anaphylaxis. On admission, routine blood tests were within normal limits and his Tolulized Red Unheated Serum Test (TRUST) value was $1: 1$. He was treated with etimicin and ornidazole for 2 days before the operation. On August 24th, the patient underwent incision and drainage for the perianal abscess. One hour after the surgery, he developed neck pain, dizziness, chest pressure, and high blood pressure up to $160 / 90 \mathrm{mmHg}$. Two hours after the surgery, the patient developed a fever up to $40^{\circ} \mathrm{C}$ with decreased blood pressure of $80 / 50 \mathrm{mmHg}$. Blood tests showed neutrophilic leukocytosis and a procalcitonin level of $66.9 \mathrm{ng} / \mathrm{mL}$ (normal value $<0.5 \mathrm{ng} / \mathrm{mL})$. After fluid resuscitation, his temperature dropped to $36.8^{\circ} \mathrm{C}$. He was then transferred to the intensive care unit (ICU). For the next 3 days, the patient was treated with intravenous imipenem-cilastatin, as well as dopamine to maintain blood pressure. Repeat blood tests showed worsening neutrophilic leukocytosis (Figure 1). The procalcitonin value was always > $200 \mathrm{ng} /$ $\mathrm{mL}$ (Figure 2). The patient developed MODS on August 27 th. On the same day, abscess culture revealed Neisseria gonorrhoeae and Staphylococcus haemolyticus (Table 1) while multiple blood cultures revealed the growth of $R$. mannitolilytica (Table 2) that was sensitive to ceftriaxone, levofloxacin, ciprofloxacin, cefepime, and piperacillin/ tazobactam. Therefore, we changed the antibiotic therapy to intravenous ceftriaxone ( $4 \mathrm{~g}$ daily) and levofloxacin $(0.5$ g daily). Six days later (September 2nd), repeat blood cultures showed no bacterial growth. The incision wound

Time-WBC curve

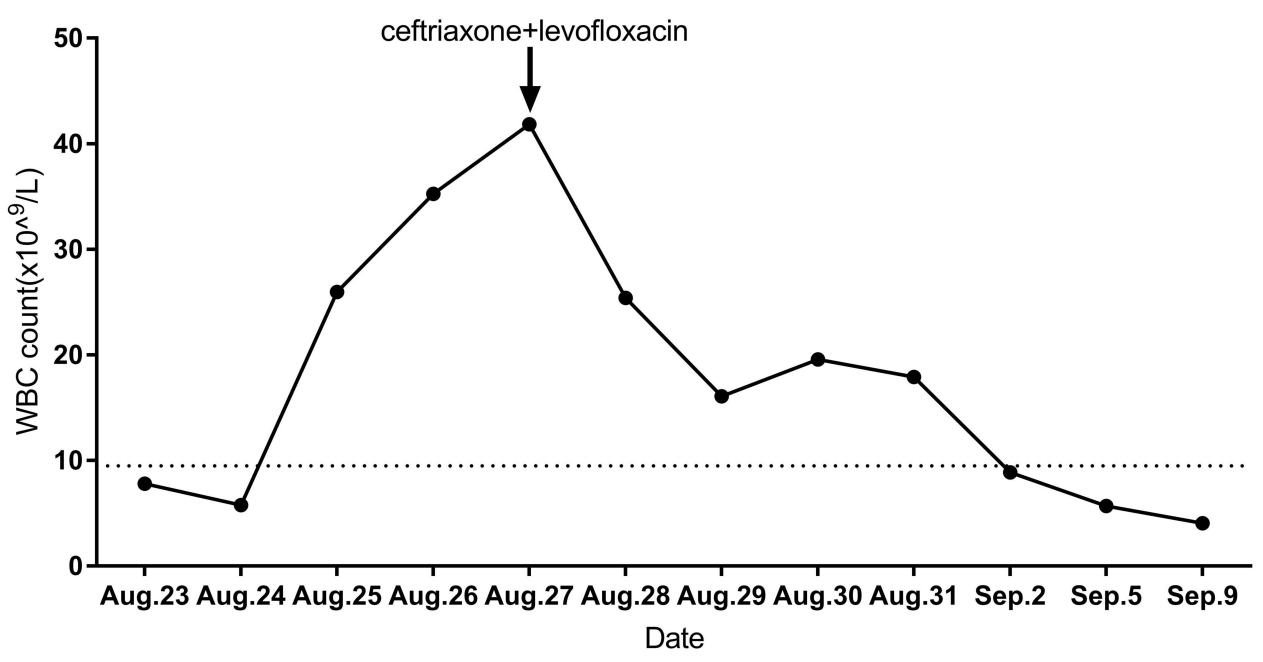

Figure I Changes in the white blood cell count during the treatment. Abbreviation: WBC, white blood cells.

Time- Lg $_{10}$ PCT curve

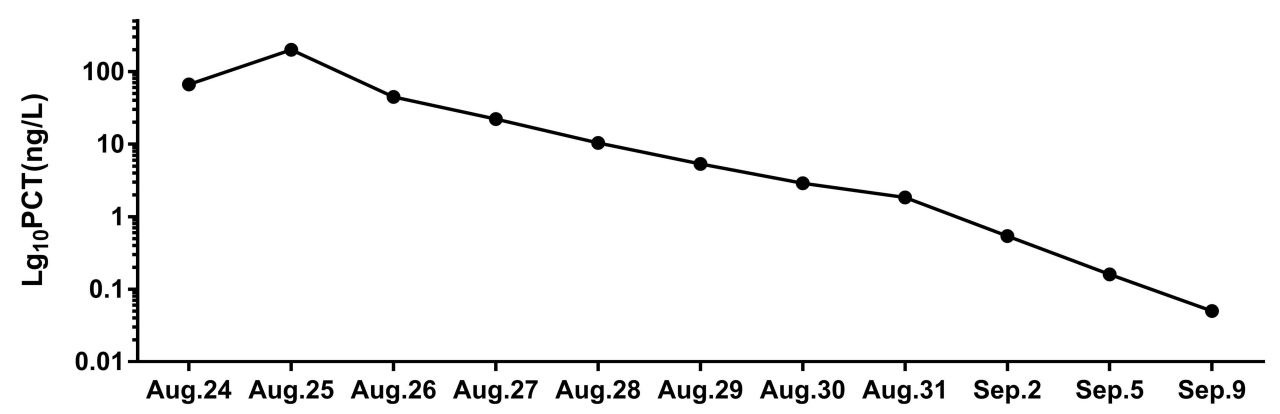

Figure 2 Changes in the concentrations of procalcitonin logarithmic values during the treatment. Abbreviation: PCT, procalcitonin. 
Table I Minimum Inhibitory Concentrations for the S. haemolyticus Isolated in This Study

\begin{tabular}{|l|c|}
\hline Antibiotic & $\begin{array}{c}\text { Minimum Inhibitory } \\
\text { Concentration }\end{array}$ \\
\hline Cefaclor & $0(\mathrm{~S})$ \\
Clindamycin & $\leq 0.25(\mathrm{~S})$ \\
Erythrocin & $\geq 8(\mathrm{R})$ \\
Ciprofloxacin & $\leq 0.5(\mathrm{~S})$ \\
Levofloxacin & $\leq 0.15(\mathrm{~S})$ \\
Oxacillin & $\leq 0.25(\mathrm{~S})$ \\
Gentamicin & $\leq 0.5(\mathrm{~S})$ \\
Rifampicin & $\leq 0.5(\mathrm{~S})$ \\
Quinupristin/Dalfopristin & $\leq 0.25(\mathrm{~S})$ \\
Tigecycline & $\leq 0.15(\mathrm{~S})$ \\
Trimethoprim/ & $\leq 10(\mathrm{~S})$ \\
sulfamethoxazole & \\
Cefamandole & $0(\mathrm{~S})$ \\
Moxifloxacin & $\leq 0.5(\mathrm{~S})$ \\
Penicillin-G & $\leq 0.0(\mathrm{R})$ \\
Tetracycline & $\leq \mathrm{I}(\mathrm{S})$ \\
Vancomycin & $\leq 0.5(\mathrm{~S})$ \\
Linezolid & $\mathrm{I}(\mathrm{S})$ \\
Cefoxitin screening & $\mathrm{Neg}(-)$ \\
Clindamycin induction test & $\mathrm{Neg}(-)$ \\
\hline
\end{tabular}

Note: Minimum inhibitory concentration was interpreted according to the Clinical and Laboratory Standards Institute (CLSI) criteria for Staphylococcus spp. Abbreviations: R, resistant; S, susceptible; Neg, negative.

in the perianal area healed well. The patient had a satisfactory recovery with no complications during a 6-month follow-up period.

Table 2 Minimum Inhibitory Concentrations for the R. mannitolilytica Isolated in This Study

\begin{tabular}{|l|c|}
\hline Antibiotics & $\begin{array}{c}\text { Minimum Inhibitory } \\
\text { Concentration }\end{array}$ \\
\hline Aztreonam & $\geq 64(\mathrm{R})$ \\
Ceftazidime & $\geq 64(\mathrm{R})$ \\
Ciprofloxacin & $0.5(\mathrm{~S})$ \\
Ceftriaxone & $2(\mathrm{~S})$ \\
Cefepime & $4(\mathrm{~S})$ \\
Gentamicin & $\geq 16(\mathrm{R})$ \\
Imipenem & $8(\mathrm{I})$ \\
Levofloxacin & $\mathrm{I}(\mathrm{S})$ \\
Tobramycin & $\geq 16(\mathrm{R})$ \\
Trimethoprim/ & $\leq 20(\mathrm{~S})$ \\
sulfamethoxazole & $16(\mathrm{~S})$ \\
Piperacillin/tazobactam & $\geq 64(\mathrm{R})$ \\
Amikacin & \\
\hline
\end{tabular}

Note: Minimum inhibitory concentration was interpreted according to the Clinical and Laboratory Standards Institute (CLSI) criteria for non-Enterobacteriaceae. Abbreviations: R, resistant; S, susceptible; I, intermediate.

\section{Methods to Identify Pathogen}

Blood cultures were incubated in a BACT/ALERT ${ }^{\circledR}$ system (bioMérieux, France) at the Clinical Microbiology Laboratory of the University of Chinese Academy of Science Shenzhen Hospital (GuangMing), China. They were seeded on the blood and MacConkey agar plates, and incubated in a $35^{\circ} \mathrm{C}$ incubator with $5 \% \mathrm{CO}_{2}$ for 24 hours. The colony determination was performed with MALDI-TOF Mass Spectrometry (Bruker, United States), which revealed R. mannitolilytica (confidence level of $99 \%$ ).

Antimicrobial susceptibility was evaluated using the broth microdilution method (VITEK-2 CIMPACT, bioMérieux, France). Since there are no Clinical and Laboratory Standards Institute (CLSI) susceptibility breakpoints available for Ralstonia spp., the minimum inhibitory concentration (MIC) results were interpreted based on the criteria used for non-Enterobacteriaceae. As shown in Table 2, $R$. mannitolilytica was resistant to most $\beta$-lactams and aminoglycosides, but susceptible to piperacillin/ tazobactam.

\section{Discussion}

R. mannitolilytica infections have been reported worldwide in recent years, especially in immunosuppressed patients. Monica Basso ${ }^{1}$ reported a case of persistent bacteremia caused by $R$. mannitolilytica and $R$. pickettii in an ICU in 2019. Matteo Boattini ${ }^{7}$ reported $R$. mannitolilytica bacteremia in a kidney transplant recipient in 2018. There were three cases of bacteremia caused by $R$. mannitolilytica in a neonatal ICU in Curitiba, Southern Brazil, in 2013-2015. ${ }^{8}$ Between 2015 and 2018, one cardiac carcinoma patient with bacteremia caused by $R$. mannitolilytica and four patients with $R$. mannitolilytica-induced bloodstream infections were reported in China. ${ }^{9,10}$ Our case report is the first record of a patient who had bloodstream $R$. mannitolilytica infection after perianal abscess surgery.

$R$. mannitolilytica is an emerging opportunistic pathogen that is a member of the Ralstonia family. ${ }^{2}$ It is an aerobic gram-negative bacillus that is widely present in external environments, such as water, soils, and plant surfaces. ${ }^{11} R$. mannitolilytica has adapted to thrive in warm, wet, and low nutritional conditions. It can survive 5 weeks in distilled water at $4{ }^{\circ} \mathrm{C}$ and 26 weeks in distilled water at $26{ }^{\circ} \mathrm{C}$. It can also pass through a $2 \mu \mathrm{m}$ membrane filter and is resistant to antiseptics. ${ }^{12}$

R. mannitolilytica has low virulence and is therefore generally considered an opportunistic pathogen that causes 
diseases only in immunocompromised individuals. ${ }^{6}$ Our patient was a typical immunocompromised person. ${ }^{13} \mathrm{He}$ had a history of syphilis but did not receive standard treatment due to his allergy to penicillin. Long-term Treponema pallidum infections could weaken his immune system $^{14}$ and lower his cellular immunity, which led to imbalanced Th1/Th2, ${ }^{15}$ defective $\mathrm{CD} 4^{+} \mathrm{CD} 25^{-} \mathrm{T}$ cells, ${ }^{16}$ decreased $\mathrm{CD}^{+} \mathrm{T}$ cells, and reduced $\mathrm{CD} 4^{+} / \mathrm{CD}^{+}{ }^{+17}$ In addition, he was found to have gonorrhea. As the patient was under stress due to perianal abscess and the surgical incision, it was easy for him to develop sepsis due to an opportunistic pathogen such as $R$. mannitolilytica.

When immunocompromised patients are infected by $R$. mannitolilytica, the disease can progress rapidly into sepsis or even MODS if the infection is not controlled early. The selection of sensitive antibiotics should be based on the culture results. According to the literature, R. mannitolilytica is often resistant to multiple antibiotics. There are no treatment guidelines or drug susceptibility recommendation for this pathogen. In the present case, the $R$. mannitolilytica was sensitive to quinolones, third and fourth generation cephalosporins, piperacillin/tazobactam, and cotrimoxazole.

It is worth noting that postoperative sepsis is often due to the pathogen spreading from the original site of infection. We administrated aminoglycosides to this patient since Escherichia coli is a common infecting organism in the perianal area and is usually sensitive to this class of antibiotics. However, a few hours after the surgery, the patient rapidly developed a high fever, refractory low blood pressure, and anuria despite the massive fluid resuscitation. Laboratory tests also showed elevated inflammatory markers. After the patient was transferred to the ICU, he was given dopamine to maintain his blood pressure. The broad-spectrum imipenem-cilastatin was also administrated. We began to suspect a deep perianal infection and initiated a search for unusual pathogens. Subsequent pelvic magnetic resonance (MR) and computed tomography (CT) scans of the chest and abdomen did not show any deep infections or other foci of infection.

Despite treatment with antibiotics, fluid resuscitation, and vasopressors, the patient's condition continued to deteriorate. Laboratory tests showed multi-organ failure, including abnormal liver and renal functions, and elevated cardiac enzymes. Finally, his cultures demonstrated that the bacteria from the perianal pus were distinct from those in the blood culture. Sepsis in this patient was confirmed to be due to $R$. mannitolilytica. The antibiotics were changed, which resulted in a satisfactory outcome. The clinical consensus is that a combination of two types of antibiotics is required to treat $R$. mannitolilytica. We finally selected levofloxacin and ceftriaxone, which are also effective in treating infections caused by S. haemolyticus, N. gonorrhoeae, and syphilis spirochetes. It is important for clinicians to pay attention to the bacterial culture and drug sensitivity, especially in febrile patients.

The present case provided valuable experience in our clinical practice with the treatment of infections from opportunistic pathogens, such as $R$. mannitolilytica. Clinicians should pay close attention to these infections since they are rare but can cause serious outcomes due to their multidrug-resistant profiles.

\section{Ethic Approval}

This study was approved by the ethics committee of the University of Chinese Academy of Science Shenzhen Hospital (GuangMing) (LL-KT-2021237).

\section{Consent to Publish}

Written informed consent was provided by the patient to allow the case details and any accompanying images to be published.

\section{Funding}

This research was supported by the Project of Shenzhen Guangming District Science and Technology Innovation Commission (No. 2020R01122) and Guangming District Economic Development Special Fund, Shenzhen China (No. GM2019020013).

\section{Disclosure}

The authors report no conflicts of interest in this work.

\section{References}

1. Basso M, Venditti C, Raponi G, et al. A case of persistent bacteraemia by Ralstonia mannitolilytica and Ralstonia pickettii in an intensive care unit. Infect Drug Resist. 2019;12:2391-2395. doi:10.2147/idr. s206492

2. Ryan MP, Adley CC. Ralstonia spp.: emerging global opportunistic pathogens. Eur J Clin Microbiol Infect Dis. 2014;33(3):291-304. doi:10.1007/s10096-013-1975-9

3. Ryan MP, Pembroke JT, Adley CC. Differentiating the growing nosocomial infectious threats Ralstonia pickettii and Ralstonia insidiosa. Eur J Clin Microbiol Infect Dis. 2011;30(10):1245-1247. doi:10.1007/ s10096-011-1219-9

4. Adley CC, Saieb FM. Comparison of bioMérieux API 20NE and Remel RapID NF Plus, identification systems of type strains of Ralstonia pickettii. Lett Appl Microbiol. 2005;41(2):136-140. doi:10.1111/j.1472-765X.2005.01737.x 
5. Glass MB, Popovic T. Preliminary evaluation of the API 20NE and RapID NF plus systems for rapid identification of Burkholderia pseudomallei and B. mallei. J Clin Microbiol. 2005;43(1):479-483. doi:10.1128/jcm.43.1.479-483.2005

6. Vaneechoutte M, Kämpfer P, De Baere T, Falsen E, Verschraegen G. Wautersia gen. nov., a novel genus accommodating the phylogenetic lineage including Ralstonia eutropha and related species, and proposal of Ralstonia [Pseudomonas] syzygii (Roberts et al. 1990) comb. nov. Int J Syst Evol Microbiol. 2004;54(Pt 2):317-327. doi:10.1099/ ijs.0.02754-0

7. Boattini M, Bianco G, Biancone L, Cavallo R, Costa C. Ralstonia mannitolilytica bacteraemia: a case report and literature review. Infez Med. 2018;26(4):374-378.

8. Souza DC, Palmeiro JK, Maestri AC, et al. Ralstonia mannitolilytica bacteremia in a neonatal intensive care unit. Rev Soc Bras Med Trop. 2018;51(5):709-711. doi:10.1590/0037-8682-0118-2018

9. Shao HL, Wang DC, Zhang XX, et al. One case of Ralstonia solani isolated from blood specimen of a patient with cardia cancer. Chin J Clin Lab Sci. 2015;33(3):240.

10. Huang Y, Zhang ZY, Wang YT, et al. Clinical characteristics and homology analysis of 4 dialysis patients with bloodstream infections caused by Ralstonia mannitolilytica. Chin J Nosocomiol. 2018;28 (11):1658-1678.

11. Lucarelli C, Di Domenico EG, Toma L, et al. Ralstonia mannitolilytica infections in an oncologic day ward: description of a cluster among high-risk patients. Antimicrob Resist Infect Control. 2017;6:20. doi:10.1186/s13756-017-0178-z
12. Debarge R, Nicolle MC, Pinaroli A, Ait Si Selmi T, Neyret P. [Surgical site infection after total knee arthroplasty: a monocenter analysis of 923 first-intention implantations]. Rev Chir Orthop Reparatrice Appar Mot. 2007;93(6):582-587. doi:10.1016/s00351040(07)92680-x. Chinese.

13. Yang RD, Liang YH, Lin LY. The significance of the lymphocyte subsets detect in peripheral blood of syphilitic patients. Chin $J$ Microecol. 2004;4. Available from: http://en.cnki.com.cn/Article_en/ CJFDTOTAL-ZGWS200404021.htm. Accessed October 19, $202 \overline{1}$

14. Li XC, Su XH. [Immunological mechanisms of Neisseria gonorrhoeae infection: an update]. Zhonghua Nan Ke Xue. 2018;24 (5):452-456. Chinese.

15. Fitzgerald TJ. The Th1/Th2-like switch in syphilitic infection: is it detrimental? Infect Immun. 1992;60(9):3475-3479. doi:10.1128/ iai.60.9.3475-3479.1992

16. Taylor AL, Llewelyn MJ. Superantigen-induced proliferation of human CD4+CD25- T cells is followed by a switch to a functional regulatory phenotype. J Immunol. 2010;185(11):6591-6598. doi: $10.4049 /$ jimmunol.1002416

17. Roncarolo MG, Levings MK. The role of different subsets of $\mathrm{T}$ regulatory cells in controlling autoimmunity. Curr Opin Immunol. 2000;12(6):676-683. doi:10.1016/s0952-7915(00)00162-x
Infection and Drug Resistance

\section{Publish your work in this journal}

Infection and Drug Resistance is an international, peer-reviewed openaccess journal that focuses on the optimal treatment of infection (bacterial, fungal and viral) and the development and institution of preventive strategies to minimize the development and spread of resistance. The journal is specifically concerned with the epidemiology of
Dovepress

antibiotic resistance and the mechanisms of resistance development and diffusion in both hospitals and the community. The manuscript management system is completely online and includes a very quick and fair peerreview system, which is all easy to use. Visit http://www.dovepress.com/ testimonials.php to read real quotes from published authors. 